\title{
Impactos das Diferentes Formas de Mensuração da Pobreza nas Variações do Índice de Bem-estar Pessoal*
}

\author{
James Ferreira Moura Jr.** \& Jorge Castellá Sarriera \\ Universidade Federal do Rio Grande do Sul, Porto Alegre, RS
}

\begin{abstract}
RESUMO - O objetivo deste trabalho é analisar o impacto de diferentes formas de mensuração da pobreza nas variações do Índice de Bem-Estar Pessoal (BEP) de indivíduos pobres e não pobres. Foram realizadas análises comparativas com o teste $t$ de Student e Análise Multivariada de Variância (MANOVA), em uma perspectiva comparativa de diferentes formas de mensuração da pobreza com 731 pessoas, provenientes de um estado do Nordeste (57\%) e do Sul (43\%) do Brasil, com idade média de 36,68 anos ( $\mathrm{DP}=16,02)$. Utilizaram-se medidas monetárias e multidimensionais da pobreza. Identifica-se que, independente da forma de mensuração da pobreza, há um impacto negativo no BEP. Porém, observa-se que a utilização da perspectiva multidimensional tem uma maior capacidade de identificação dos impactos psicossociais da pobreza.
\end{abstract}

PALAVRAS-CHAVE: pobreza, bem-estar pessoal, abordagem multidimensional, abordagem monetária

\section{Impacts of Different Forms of Poverty Measurement on Variatons of the Personal Wellbeing Index}

\begin{abstract}
The aim of this study is to analyze the impact of different forms of poverty measurement on variations of the Personal Well-being Index (PWI) of poor and non-poor individuals. Comparative analyzes were performed with the Student's t-test and Multivariate Analysis of Variance (MANOVA) in a comparative perspective of different forms of poverty measurement. The sample consisted of 731 Brazilian subjects, $57 \%$ from a northeastern state and $43 \%$ form a southern state, with a mean age of 36,7 years $(S D=16,0)$. Monetary and multidimensional measures of poverty were used. The results indicate that, regardless of the form of poverty measurement, there is a negative impact on PWI. However, the use of the multidimensional perspective seems to have a greater capacity to identify the psychosocial impacts of poverty.
\end{abstract}

KEYWORDS: poverty, personal well being, multidimensional approach, monetary approach

Entende-se que a forma utilizada na conceituação e mensuração da pobreza pode ter impacto significativo na quantidade de pessoas concebidas, como nessa situação e na identificação da influência de outros fatores relacionados a esse fenômeno. Isso ocorre porque a pobreza tem múltiplas perspectivas de medição e conceituação (Feres \& Villatoro, 2013). Concebe-se também que aspectos vinculados à dimensão subjetiva não são tratados como prioritários nas investigações (Samman, 2007).

De acordo com Moura Jr. e Sarreira (2017), as formas de definição da pobreza são diversas, mas elas não necessariamente se vinculam às estratégias de mediação utilizadas nas investigações. Portanto, há desde perspectivas mais críticas de conceituação da pobreza, como vinculada à desigualdade social e como ferramenta de manutenção da realizada social hierarquizada (Abranches, Santos, \& Coimbra, 1987; Cidade, Moura Jr., \& Ximenes, 2012; Cidamore, \& Cattani, 2007; Góis, 2005). Também, existem conceituações que concebem a pobreza como um estado de injustiça social, baseado em uma forma de vida privada de liberdade (Comim, 2005, 2008; Sen, 1980, 2000; Nussbaum \& Sen, 1996). Igualmente, há concepções de pobreza somente baseadas na deficiência monetária, sendo esvaziadas de articulações vinculadas à realidade social (Barros, Carvalho, \& Franco, 2007; Laderchi, Saith, \& Stewart, 2003).

Já as diferentes formas de mensuração da pobreza podem estar situadas em duas grandes perspectivas. De acordo com o Relatório do Desenvolvimento Humano de 1997, do Programa das Nações Unidas, há geralmente a perspectiva

\footnotetext{
* Apoio: CAPES

** E-mail: jamesferreirajr@gmail.com

- Submetido: 27/10/2015; Revisado: 14/10/2017; Aceito: 16/02/2018.
} 
conglomerativa e distributiva nas mensurações da pobreza (Programa das Nações Unidas para o Desenvolvimento [PNUD], 1997). A primeira representa a pobreza abordada como insuficiência de renda, em que o bem-estar social estaria situado unicamente como uma medida econômica. Nessa perspectiva, encontram-se as linhas de pobreza, sendo usadas usualmente nas investigações, nas políticas públicas e nos organismos internacionais. Elas se baseiam em um mínimo financeiro relacionado à sobrevivência (PNUD, 1997). A forma de medição distributiva se refere à pobreza amparada por indicadores multidimensionais com aspectos qualitativos, comparativos e voltados para uma compreensão específica de desenvolvimento humano (Comim \& Bagolin, 2002).

De acordo com Accorssi, Scarparo e Guareschi (2012), essas perspectivas monetárias unidimensionais sofrem muitas críticas, porque reduzem a questão nutricional de subsistência, convertidas em dinheiro às únicas necessidades do indivíduo. Segundo o Instituto de Pesquisa Econômica Aplicada (IPEA, 2014), há uma linha de pobreza utilizada mundialmente, desenvolvida pelo Banco Mundial, de US\$ 1,25 dólares de Paridade de Poder de Compra (PPP) por pessoa. Critica-se a ocorrência dessa mesma forma de mensuração da pobreza em países com maiores e menores níveis de renda (Feres \& Villatoro, 2013). Dessa maneira, é imperativa a necessidade de formas de mensuração da pobreza que ultrapassem essa lógica redutora desse fenômeno a questões econômicas.

Compreende-se que a Abordagem das Necessidades Humanas Básicas foi extremamente significativa no avanço das formas de medição da pobreza, pois introduziu o modelo multidimensional de encarar esse fenômeno, como também repercutiu na criação do Índice de Desenvolvimento Humano (IDH) usado pela Organização das Nações Unidas para medir a qualidade de vida dos países. Este Índice foi desenvolvido para ser de fácil mensuração, aplicabilidade e interpretação, abarcando escolhas econômicas e sociais (Bagolin \& Comim, 2008).

Apesar desses avanços, ele abrange, ainda de forma restrita, a complexidade do fenômeno pobreza, por estar somente constituído das dimensões saúde, educação e rendimentos. Aponta-se criticamente que as necessidades básicas estão interligadas, ou seja, não são independentes como apontadas no Índice (PNUD, 2010). Além desse instrumento, há outros indicadores multidimensionais da pobreza: o Índice de Pobreza Humana (IPH), o IDH Ajustado à Desigualdade (IDHAH) e o Índice de Pobreza Multidimensional (IPM). O IPH é uma derivação do IDH, mas está relacionado à mensuração da pobreza a partir da perspectiva multidimensional, identificando a acumulação das privações existentes. São utilizadas como variáveis a longevidade pela parcela da população que vive menos de quarenta anos, o conhecimento pela quantidade de analfabetos e o padrão de vida pela porcentagem de indivíduos que não acessam serviços de saúde e de abastecimento de água potável, assim como de crianças subnutridas. Apesar de o IPH representar um avanço ao IDH, as críticas são as mesmas relacionadas ao Índice de Desenvolvimento Humano.

O Índice de Desenvolvimento Humano Ajustado à Desigualdade (IDHAH) tem como objetivo a identificação da desigualdade de cada dimensão do IDH nos países. Apesar de representar novamente um progresso, devido a problemas técnicos, não há como identificar se indivíduos sofrem uma ou várias privações. O IPM é o único, até então, que realmente representa uma significativa melhoria na mensuração do caráter multidimensional da pobreza em relação aos outros índices. Ele identifica as privações específicas dos indivíduos e das famílias, sendo uma escala construída de forma consensual internacionalmente e elaborada a partir de experiências em comunidades pobres. Possui dimensões semelhantes ao IDH, como saúde, educação e padrão de vida, mas expande essas dimensões em 10 indicadores, que são: ativos, pavimento, eletricidade, água, sanitários, combustível da cozinha, crianças matriculadas na escola, anos de escolaridade, mortalidade infantil e nutrição (PNUD, 2010).

No entanto, esse último índice ainda possui algumas limitações. Por exemplo, não verifica de forma detalhada os anos de estudo de cada indivíduo, delimitando como ponto de inclusão da pobreza somente situar-se abaixo de 5 anos de escolaridade e não possuir criança em idade escolar na escola. Esse índice não abrange questões relacionadas ao trabalho e à renda do indivíduo e da sua família. Além disso, somente traz questões relacionadas à saúde voltadas à subnutrição e à morte de criança. Por fim, não traz indicadores relacionados aos aspectos psicológicos da pobreza e não abrange questões referentes à liberdade, à autonomia e à participação social do sujeito (PNUD, 2010). Além disso, ele funciona bem para populações residentes no continente africano, mas para os países latinos americanos esse instrumento não tem uma boa capacidade preditiva da pobreza multidimensional. Isso ocorre porque os pontos de corte estão relacionados às situações já superadas de forma geral na América Latina, como a mortalidade infantil, a desnutrição, ausência de energia elétrica e de vaso sanitário nas residências (Feres \& Villatoro, 2013).

Portanto, os instrumentos utilizados por grandes organizações mundiais para mensuração da pobreza têm também influência nas pesquisas desenvolvidas sobre o tema. É necessário, então, refletir sobre a necessidade de formas de medição mais precisas. Compreende-se que é necessária a centralidade dos preceitos da Abordagem das Capacitações (AC) para que isso ocorra. Essa abordagem se baseia na compreensão de pobreza atrelada ao desenvolvimento humano, que é constituído desde a promoção das necessidades fisiológicas, até o fortalecimento de aspectos psicológicos de liberdade e de autonomia. Dessa maneira, essa abordagem situa-se em uma posição de questionamento da perspectiva monetária da pobreza (Sen, 2000). 
Os seres humanos são abordados como portadores de funcionamentos que são os estados e as atividades desempenhadas. Ou seja, esses funcionamentos são as diferentes formas de ser e de fazer realizadas pelo indivíduo a partir dos recursos disponíveis. O conjunto de funcionamentos é entendido como capacitações que representam a liberdade da pessoa de escolher funcionamentos que sejam importantes para si (Sen, 1996). Então, a pobreza representa a privação de capacitações básicas provenientes da falta de liberdade de eleição de funcionamentos valiosos para os indivíduos. Essa falta de liberdade pode estar relacionada a uma situação econômica adversa, uma política pública não efetiva ou uma realidade social opressora e violenta (Cohen, 1996).

Apesar desse avanço, salienta-se a necessidade de investigar questões subjetivas na perspectiva multidimensional da pobreza (Chiappero-Martinetti, 2000). Aponta-se que esses estudos podem ser abordados como controversos, porque utilizam parâmetros não objetivos de mensuração. Apesar dessas críticas, compreende-se que há necessidade de articulação de aspectos objetivos - por meio da normatização de funcionamentos e capacitações concretos na avaliação do bem-estar humano - e subjetivos (Comim, 2005). Defendendo esses últimos aspectos, Sen (1996) enfatiza que o bem-estar representaria a capacitação para escolher com liberdade diferentes formas de vida importantes para o indivíduo. Assim, entende-se que, quando há a utilização conjunta da Abordagem das Capacitações e a conceituação de bem-estar subjetivo para o entendimento do impacto da pobreza no bem-estar humano, alcança-se um nível de compreensão da realidade de pobreza que abarca fatores objetivos e subjetivos importantes para o entendimento desse fenômeno.

Observa-se que geralmente as investigações sobre os impactos da pobreza no bem-estar utilizam o conceito e medida do bem-estar subjetivo. Diener (2012) define bem-estar subjetivo como avaliações cognitivas e afetivas relacionadas à vida de forma geral. Nessas avaliações, estão presentes julgamentos vinculados à satisfação, como também reações emocionais positivas e negativas aos acontecimentos vividos. De acordo com Moura Jr. e Sarriera (2017), o bem-estar subjetivo geralmente é mensurado pela Escala de Satisfação com a Vida (Satisfaction with Life Scale), desenvolvida por Diener, Emmons, Lasen e Griffin (1985). É importante também salientar que o bem-estar subjetivo é abordado como uma das dimensões "perdidas" da pobreza, sendo apontada a necessidade de sua maior investigação. A partir da Abordagem das Capacitações, observa-se que o bem-estar subjetivo é concebido como um exercício de liberdade quando o indivíduo tem a possibilidade de realizar uma avaliação global de sua vida. (Samman, 2007).

Há uma série de estudos e pesquisas que almejam identificar a relação da pobreza e do bem-estar subjetivo. No entanto, de acordo com Hochman e Skopeck (2013), a privação da renda é utilizada como principal indicador da pobreza na maioria das investigações realizadas. Igualmente, existem várias formas de compreensão dessa relação. Em um estudo com 19 países, foi identificado que pessoas em situação de riqueza tinham um maior nível de bem-estar subjetivo que pessoas em situação de pobreza (Eastearlin, 1995). Em uma investigação com pessoas em situação de extrema pobreza nos Estados Unidos, também foram apontados baixos níveis de bem-estar subjetivo nesse público pesquisado (Biswas-Diener \& Diener, 2006).

No entanto, há alguns paradoxos que incidem sobre essa relação entre pobreza e bem-estar subjetivo. Há o paradoxo de Eastearlin, ou Paradoxo da Felicidade, que concebe que existe um limite para o impacto da renda no bem-estar subjetivo. Geralmente, em populações com altos índices de riqueza, não há predição positiva da renda no aumento do bem-estar subjetivo (Eastearlin 2000). De forma inversa, há o paradoxo dos Camponeses Felizes e dos Milionários miseráveis (paradox of happy peasants and miserable millionaires), que indica que as pessoas em situação de pobreza podem também apresentar maiores níveis de bem-estar subjetivo que indivíduos em situação de riqueza (Graham, 2009).

A partir desses resultados contrastantes, deve-se compreender que o bem-estar subjetivo é uma medida de avaliação abstrata de satisfação com a vida, pois é uma escala de âmbitos livre de contextos. Dessa maneira, é necessário utilizar uma categoria e uma forma de mensuração que esteja vinculada aos aspectos concretos da vida, porque se concebe que a pobreza é constituída de aspectos concretos vinculados a uma realidade de privação. Assim, o bemestar pessoal (BEP) poderia ser considerado esse conceito, porque parte de avaliações subjetivas e objetivas (Ribeiro \& Cummins, 2008). Dessa maneira, o bem-estar pessoal é construído de aspectos referentes a julgamentos cognitivos sobre a vida, contemplando aspectos afetivos e concretos ligados a diferentes âmbitos, como saúde, relações pessoais, segurança, futuro, vinculação comunitária e padrão de vida (Cummins et al., 2003).

No entanto, dependendo da perspectiva de mensuração da pobreza, há uma variabilidade da sensibilidade do instrumento, no tocante aos impactos negativos desse fenômeno no bem-estar. Ainda, é necessário ressaltar que muitas investigações envolvendo pobreza e bem-estar estariam relacionadas somente a aspectos monetários e unidimensionais da pobreza (Moura Jr., Cidade, Ximenes, \& Sarriera 2014; Moura Jr. \& Sarriera, 2017). Dessa maneira, é premente o desenvolvimento de pesquisas que abordem também indicadores multidimensionais da pobreza e seus impactos no bem-estar pessoal de indivíduos inseridos nessa realidade de privação. Além disso, aponta-se a necessidade de realizar mais estudos sobre o Índice de Bem-Estar Pessoal em países com significativos índices de pobreza (Smyth, Nielsen, \& Zhai, 2010). Com isso, este estudo tem como objetivo analisar o impacto, de diferentes formas, da mensuração da pobreza, nas variações do Índice de bemestar pessoal de indivíduos pobres e não pobres de dois estados brasileiros. 


\section{MÉTODO}

Será utilizado o delineamento de pesquisa descritivo correlacional, de caráter transversal e baseado em observações (enquete) (Fowler Jr., 2011), ou também conhecido como levantamento. Esse delineamento se refere a uma investigação de forma padronizada, relacionada a temáticas específicas da pesquisa com uma amostra por conveniência da população estudada (Robson, 1993). Este estudo é um recorte de uma pesquisa interinstitucional desenvolvida por duas universidades brasileiras, em territórios com alta incidência de pobreza nos seus respectivos estados.

\section{Participantes}

A amostra é composta por 731 pessoas, com idade média de 36,68 anos $(D P=16,02)$, sendo $72,2 \%$ mulheres. Sobre as regiões onde foi realizada essa investigação, $57 \%$ dos participantes residem em um dos estados do Nordeste, enquanto $43 \%$ deles moram em um estado do Sul do país. É importante salientar que essa pesquisa foi desenvolvida em duas cidades de cada estado, ocorrendo em bairros com altos índices de vulnerabilidade social. Relacionado à escolaridade, 5,3\% são analfabetos; 19\% tem Ensino fundamental incompleto; $16 \%$ possuem Ensino Fundamental Completo; $15,2 \%$ portam o Ensino Médio e 44,5\% o Ensino Médio Completo. É evidenciado que 57,9\% não exerciam atividade remunerada e $74,8 \%$ recebiam até um salário mínimo no período de realização da investigação.

Os critérios utilizados para participação da investigação foram: ser residente nos bairros com altos índices de vulnerabilidade social escolhidos para a realização da pesquisa; possuir a idade mínima de 18 anos para assumir a responsabilidade de participação na pesquisa e declarar anuência, a partir do conhecimento das condições de pesquisa e da assinatura do termo de consentimento livre e esclarecido. Com esses critérios, a amostra foi dividida internamente, a partir de grupos caracterizados pela situação de pobreza e fora dessa situação, por meio das diferentes formas de mensuração desse fenômeno, como indicado no tópico de análise. É importante salientar que essa divisão foi somente realizada após a coleta e análise de dados, não tendo sido utilizada como critério de participação na investigação.

\section{Instrumentos}

É utilizado o Índice de Bem-Estar Pessoal (Personal Wellbeing Index), que foi primeiramente aplicado na Austrália, tendo, posteriormente, difusão mundial (Cummins et al., 2003). Esse índice tem o objetivo de mensurar a satisfação global com a vida, a partir de âmbitos específicos, portando significativos índices de validade, replicabilidade e composição, como o Alfa de Cronbach, de 0,85 (Ribeiro
$\&$ Cummins, 2008). Neste estudo, a escala tem um Alfa de Cronbach de 0,84. Os âmbitos utilizados são padrão de vida, saúde pessoal, realizações, relações pessoais, segurança, relações comunitárias e segurança quanto ao futuro. Esses indicadores são avaliados a partir de um ranking de resposta de 0 (totalmente insatisfeito) até 10 (totalmente satisfeito; International Wellbeing Group, 2006).

Foram também utilizadas várias formas de mensuração da pobreza para avaliar o impacto dessas diferentes perspectivas no Bem-Estar Pessoal. Primeiramente, foi usada a variável monetária: "Qual o valor aproximado da sua renda pessoal mensal?". Essa variável foi usada para caracterizar o grupamento com pobreza monetária. Também, foi utilizado o IPM, desenvolvido pelo Programa das Nações Unidas para o Desenvolvimento (PNUD, 2010). Esse índice está constituído de três dimensões de privação relacionadas: (1) Ao padrão de vida (não possuir eletricidade na residência, sem água potável, sem saneamento básico, utilização de carvão ou madeira para cozinhar, possuir casa com o piso de terra, possuir no máximo um bem durável); (2) Educação (possuir todos os membros da família com menos de 5 anos de estudo e existência de pelo menos uma criança em idade escolar fora da escola) e (3) Saúde (existência de pelo menos um membro da família com desnutrição e ocorrência de falecimento de pelo menos uma criança), ampliando, assim, a compreensão da pobreza para além da perspectiva monetária.

Foi desenvolvido também o Instrumento de Mensuração da Pobreza Multidimensional (IMPM). Como base para desenvolvimento desse índice, foram utilizadas as seguintes pesquisas: Indicadores de Pobreza Multidimensional e Extrema Pobreza para Porto Alegre (Avila, Bagolin, \& Comim, 2012) e Pobreza multidimensional aplicada na África do Sul (Qizilbash \& Clark, 2005), na Itália (Chiappero-Martinetti, 2000) e na Bélgica (Lelli, 2001). Assim, para conseguir mensurar indicadores relacionados à pobreza multidimensional de forma complexa, nesse índice é indicada a utilização da metodologia dos conjuntos fuzzy, que se refere a diferentes graus de realização dos indicadores investigados (Chiappero-Martinetti \& Moroni, 2007).

A pobreza e o bem-estar são construtos considerados difíceis de serem identificados em sua complexidade e totalidade, sendo essa teoria geralmente utilizada para a compreensão abrangente desses fenômenos (Lelli, 2001; Qizilbash \& Clark, 2005). Dessa maneira, os indicadores e as variáveis que os constituem são padronizadas a partir de um ranking que varia de 0 , considerado o valor sem pobreza multidimensional, e 1, considerado o valor com pobreza multidimensional integral.

Assim, as variáveis dicotômicas estão relacionadas aos valores 0 (não pobre) e 1 (pobre), podendo ser concebidas a partir de uma função linear em que os valores são equidistantes e diretamente proporcionais entre os elementos 
existentes. Dessa maneira, a metodologia dos conjuntos fuzzy substitui a função bem delineada (set crisp) em que os indicadores somente estariam relacionados com os valores 0 (não-membros) ou 1 (membro). As variáveis ordinais terão valores intermediários equidistantes atribuídos entre o intervalo [0,1], podendo ser abordados a partir de uma função linear (Lelli, 2001). Então, tanto em cada variável, como em cada dimensão, quanto mais os valores das respostas estiverem próximos do 1 , serão considerados mais privados, ou seja, mais pobres e, quanto mais os valores estiverem próximos do 0 , serão considerados menos privados, ou seja, menos pobres multidimensionais.

A partir da apresentação de como é estruturada a lógica do IMPM, deve-se igualmente explicitar as dimensões que são utilizadas, definir os indicadores de cada dimensão, estipular os pontos de corte para identificação da situação de pobreza e agregar as dimensões em um índice (Feres, \& Villatoro, 2013). Dessa maneira, as dimensões e suas respectivas variáveis presentes no instrumento são apresentadas conforme a posição dos itens de resposta no ranking do índice de mensuração da pobreza multidimensional:

Educação: anos de escolaridade, que possui cinco opções de resposta: 1 = Não estudou; $0,75=$ Ensino Fundamental Incompleto; 0,5 = Ensino Fundamental Completo; 0,25 = Ensino Médio Incompleto; $0=$ Ensino Médio Completo (PNUD, 2010); adulto na família com mais de 9 anos de estudo, que possui opção de resposta dicotômica: $1=$ Sem adulto com mais de 9 anos de estudo; $0=$ Com adulto com mais de 9 anos de estudo (PNUD, 2010).

Habitação: propriedade da residência, possui quatro opções de resposta: $1=$ Ocupação; $0,66=$ Cedida; $0,33=$ Alugada; $0=$ Própria criada para a pesquisa (Desenvolvida para o IMPM)]; tipo de residência, possui quatro opções de resposta: 1 = Papelão, plástico ou compensado; $0,66=$ Taipa; 0,33 = Madeira; $0=$ Alvenaria (Qizilbash \& Clark, 2005)]; existência de água encanada, com opção de resposta dicotômica: 1 = Sem água encanada; $0=$ Com água encanada (Pnud, 2010; Qizilbash \& Clark, 2005)]; tipo de piso da casa, com três opções de resposta: $1=$ Terra batida; $0,5=$ Cimento; 0 = Cerâmica (PNUD, 2010); tipo de combustível utilizado, possui variável categórica:três opções, adaptada do PNUD (2010); existência de eletricidade, com opção de resposta dicotômica: $1=$ Com eletricidade; $0=$ Sem eletricidade (PNUD, 2010); tipo de sanitário, possui quatro opções de resposta: $1=$ sem banheiro; $0,66=$ buraco no chão; $0,33=$ vaso sanitário sem descarga; $0=$ vaso sanitário com descarga (PNUD, 2010; 2005)]; saneamento básico, com três opções de resposta: $1=$ Céu Aberto; $0,5=$ Fossa; $0=$ Sistema de Esgoto; (PNUD, 2010)] e quantidade de utensílios domésticos, que possui opção de resposta dicotômica: $1=$ Com mais de 5 utensílios domésticos; $0=$ Com menos de 5 utensílios domésticos (PNUD, 2010].

Trabalho/renda: status laboral, com opção de reposta dicotômica: 0 = empregado; 1 = desempregado (Comim et al., 2007); renda pessoal, possui cinco opções de resposta:
$1=0$ até $\mathrm{R} \$ 77,00 ; 0,75=\mathrm{R} \$ 78,00$ até $\mathrm{R} \$ 154,00 ; 0,5=\mathrm{de}$ 155,00 até $\mathrm{R} \$ 339,00 ; 0,25=\mathrm{R} \$ 339,00$ até $\mathrm{R} \$ 678,00 ; 0$ $=$ Mais de $\mathrm{R} \$ 678,00$ (Comim et al., 2007)]; renda familiar, com seis opções de resposta: $1=$ Sem rendimento; 0,75 $=$ Menos de 1 salário mínimos; $0,5=$ entre 1 e 2 salários mínimos; 0,25 = entre 3 e 4 salários Mínimo; $0=$ mais de 4 salários mínimos (Comim et al., 2007); vender algo para poder comer, possui opção de resposta dicotômica: $0=$ Não precisou vender para poder comer; $1=$ precisou vender para poder comer (Comim et al., 2007); comportamento de pedir dinheiro para poder comer, com opção de reposta dicotômica: 0 = Não pediu dinheiro para poder comer; 1 = Pediu dinheiro para poder comer (Comim et al., 2007).

Saúde: obtenção de atendimento médico, com opção de resposta dicotômica: $0=$ conseguiu atendimento médico; 1 = Não conseguiu atendimento médico (Qizilbash \& Clark, 2005; Lelli, 2001); impossibilidade de atendimento médico por falta de dinheiro, também com opção de resposta dicotômica: $0=$ Conseguiu atendimento mesmo sem dinheiro; 1 = Não conseguiu atendimento por falta de dinheiro (Desenvolvida para o IMPM); existência de mortalidade infantil, possui opção de resposta dicotômica: $0=$ Nenhuma criança morreu no domicílio; 1 = Alguma criança morreu no domicílio (PNUD, 2010); quantidade de refeições diárias, com quatro opções de resposta: $1=$ Nenhuma; 0,66 = Uma; 0,33 = Duas; $0=$ Três ou Mais (Comim et al., 2007) e existência de alguma pessoa com desnutrição, possui opção de resposta dicotômica: $1=$ existência de pessoa com desnutrição; $0=$ inexistência de pessoa com desnutrição (PNUD, 2010).

Aspectos subjetivos da pobreza: autopercepção de pobreza, com três opções de resposta: $1=$ Pobre; $0,5=$ Nem rico nem pobre; $0=$ Rico (Comin et al., 2007) e vergonha da pobreza, com opção de resposta dicotômica: $1=$ sentimento de vergonha por ser pobreza; $0=$ não sentimento de vergonha por ser pobre.

Então, há gradações do nível de pobreza multidimensional, configurando a metodologia de conjuntos fuzzy (Lelli, 2001). De acordo com Qizalbash e Clark (2005), muitos estudos que abordam a perspectiva multidimensional de pobreza não utilizam um ponto de corte para identificar um estado de maior privação. Compreende-se a situação de pobreza como diferentes gradações, sendo quanto mais próximo de 1 , maior estado de pobreza multidimensional e quanto mais próximo de 0 , menor estado de pobreza multidimensional. Assim, utilizando a estratégia realizada por Comim (2008), será desenvolvida uma média do Instrumento de Mensuração da Pobreza Multidimensional, a partir da soma dos indicadores pesquisados e sua divisão pela quantidade de indicadores. Essa média representaria a agregação horizontal das privações identificadas com a obtenção da média das dimensões pesquisadas com todos os funcionamentos para uma pessoa (Comim, 2005). Assim, todos os indicadores ou funcionamentos escolhidos não têm diferença de peso, sendo igualmente importantes para mensuração. 


\section{Procedimentos}

A investigação foi realizada nas capitais e em uma cidade do interior de cada estado participante. A equipe de pesquisa identificava os bairros com maior vulnerabilidade em cada cidade e entrava em contato com os agentes comunitários, profissionais dos Centros de Referência de Assistência Social e de Organizações Não-governamentais para levar a equipe de pesquisa nesses territórios. Eram escolhidas de forma aleatória residências com infraestrutura precária, havendo o convite para uma pessoa da casa para participar da entrevista. $\mathrm{O}$ questionário foi aplicado geralmente por meio de contato individual. Quando o participante solicitava, ele era lido pelo entrevistador. Como forma de assegurar a proteção dos direitos e da dignidade dos participantes, foi elaborado termo de consentimento livre, contando a explicitação das informações necessárias para realização da pesquisa (Barker, Pistrang, \& Elliot, 1994). É importante salientar que a pesquisa foi submetida à avaliação do comitê de ética de uma instituição pública de Ensino Superior, tendo parecer favorável à sua realização.

\section{Análises}

Análises comparativas foram desenvolvidas. Foi realizado diferentes Teste $t$ de Student para amostras independentes, comparando a média total do Índice de BEP a partir das diferentes formas de mensuração da pobreza e utilizando o nível de significância de 0,05 . Primeiramente, o BEP foi comparado em uma perspectiva de pobreza monetária, a partir de grupos de indivíduos com e sem pobreza monetária. Esses foram formados por meio da linha política administrativa de pobreza, desenvolvida a partir do Plano Brasil sem Miséria do Governo Federal, sendo $\mathrm{R} \$ 154,00$ reais por mês por pessoa (Brasil, 2014). Assim, foram criados dois grupos da amostra pesquisada: com e sem pobreza monetária, a partir da linha de pobreza brasileira.

Em seguida, a mesma média geral do Índice de BEP foi comparada com os indivíduos que são e não são considerados pobres multidimensionais pelo IPM do PNUD
(2010). De acordo com o Programa das Nações Unidas para Desenvolvimento Humano (2010), cada dimensão teria uma pontuação de 3,3, porque o valor máximo de privação é 10 . Assim, por exemplo, para as dimensões educação e saúde, como cada uma tem dois itens, os valores para cada opção de reposta é 1,67. Para a dimensão padrão de vida, cada item é atribuído o valor de 0,56 , porque ela é constituída de seis itens. Segundo diretrizes das Nações Unidas, o indivíduo é considerado pobre se tiver uma pontuação entre 3 e 10 de acordo com diferentes privações nas dimensões saúde, educação e padrão de vida PNUD (2010). Dessa maneira, foram criados um grupo com pobreza multidimensional e um grupo sem pobreza multidimensional, a partir desse critério do IPM. A média total do BEP foi comparada a partir desses dois diferentes grupos.

Por fim, é realizada uma comparação da média total do BEP a partir do IMPM. Foi realizada uma análise dos percentis das médias totais do IMPM. É importante salientar que a amostra, de forma geral, está com nível moderado de pobreza multidimensional, porque não há nenhum participante com a média do IMPM igual a 0. Assim, escolhe-se analisar os grupos com mais e menos pobreza multidimensional na amostra. A partir do percentil de 50\% do IMPM, que se refere ao valor 0,24 , foram criados dois grupos: pobres multidimensionais, com média do IMPM acima de 0,24 e não pobres multidimensional, com essa média abaixo desse valor estipulado do IMPM.

Também, foram comparados os itens do Índice de Bem Estar Pessoal por meio da Análise Multivariada de Variância (MANOVA). Os itens foram considerados variáveis dependentes a partir da comparação com os mesmos grupos apresentados anteriormente relacionados à pobreza monetária, à pobreza multidimensional do PNUD e à pobreza multidimensional do IMPM. Foi utilizado o contraste polinomial nessa análise multivariada. É importante salientar que os pré-requisitos para realizar essa análise multivariada foram contemplados, como o tamanho amostral, a relação teórica entre as variáveis dependentes, a normalidade multivariada e a homogeneidade de variância (Hair, Black, Babin, Anderson, \& Thatam, 2009).

\section{RESULTADOS}

\section{Comparações das Médias Totais BEP entre os Grupos de Pobreza Monetária, de Pobreza Vinculada ao PNUD e de Pobreza Vinculada ao IMPM}

Primeiramente, foi realizado um teste $t$ de Student para comparar a média total do Índice de BEP com o grupo de pessoas que estavam em situação de pobreza monetária e com aqueles que não estavam nessa situação. Foi identificado que há uma diferença significativa entre os dois grupos $[t(435)=-2,71, p=0,007]$, sendo que o grupo com pobreza monetária tem média mais baixa no Índice de BEP ( $M$ pobre monetário=7,07, $D P=1,99)$ que o grupo sem pobreza monetária ( $M$ não pobre monetário=7,48, $D P=1,76)$. Também, foram realizadas comparações do Índice de BEP com o grupo que não está em situação de pobreza multidimensional e o grupo que está em situação de pobreza multidimensional, segundo a medida do PNUD. Dessa maneira, identifica-se que há diferenças significativas entre os grupos $[t(724)=-3,44, p=0,001]$, sendo que o grupo com pobreza multidimensional do PNUD (Mpobre multi PNUD=7,18, DPpobre multi PNUD=1,86) tem média mais 
baixa do Índice do BEP do que o grupo sem pobreza (Mnão pobre multi $\mathrm{PNUD}=7,70, D P$ não pobre multi $\mathrm{PNUD}=1,87$ ).

Igualmente, foram realizadas comparações do BEP com os grupos de pobreza multidimensional, baseadas no IMPM. Assim, observa-se que há diferenças significativas $[t(587)=-$ $11,86, p<0,001]$ no grupo com pobreza multidimensional em relação ao grupo sem essa situação de privação. Esse último grupo ( $M$ não pobre multi IMPM=8,10, $D P$ não pobre multi IMPM=1,23) tem uma média mais alta do Índice de Bem Estar Pessoal do que as pessoas em situação de maior pobreza multidimensional ( $M$ pobre multi IMPM=6,59, $D P$ pobre multi IMPM=2,10).

\section{Comparações dos Itens do BEP entre os Grupos de Pobreza Monetária, Pobreza Multidimensional PNUD e Pobreza Multidimensional IMPM Realizadas por MANOVA}

Apresentam-se as médias dos itens do Índice de Bem Estar Pessoal a partir das diferentes formas de mensuração da pobreza, na Tabela 1 com a amostra total:

$\mathrm{Na}$ amostra total, quando se compara os grupos somente em situação de pobreza monetária, foram encontradas diferenças significativas na interação das variáveis do Índice de BEP $[F(7,722)=1,51, p=0,158$, Wilks' Lambda $=0,985]$. Comparando precisamente as diferenças a partir de cada variável, identifica-se que "satisfação com o nível de vida" $[F(1,728)=8,62, p=0,003] \mathrm{e}$ "com as coisas que tem conseguido na vida" $[F(1,728)=4,19, p=0,041]$ apresentam diferenças significativas entre os grupos pesquisados. Assim, o grupo sem pobreza monetária tem uma maior satisfação relacionada às coisas que conseguiu na vida do que aqueles com pobreza monetária. Essa mesma perspectiva ocorre com a variável satisfação com o nível de vida.

Também, foram realizados comparações dos itens do BEP com os grupos em situação de pobreza multidimensional, segundo o PNUD, na amostra total a partir da MANOVA, sendo identificado que há diferenças significativas nas interações das variáveis entre o grupo com pobreza multidimensional e sem pobreza multidimensional do $\operatorname{PNUD}[F(7,718)=3,72, p=0,001$, Wilks' Lambda $=0,965]$. Assim, as variáveis que tiveram diferenças significativas foram: "satisfação com seu nível de vida" $[F(1,724)=$ $5,82, \mathrm{p}=0,016]$; "satisfação com as coisas que você tem conseguido na vida" $[\mathrm{F}(1,724)=14,33, \mathrm{p}<.001]$; "satisfação com a sensação de estar seguro" $[F(1,724)=9,25, p=0,002]$; "satisfação com a comunidade" $[F(1,724)=8,20, p=0,004]$ e "satisfação com a segurança a respeito do futuro" $[F(1,724)=15,19, p<0,001]$.

Relacionado ao IMPM, foi identificado que há diferença significativa $[F(7,723)=29,56, p<0,001$, Wilks' Lambda $=0,777)$ na interação dos itens do BEP na amostra total, a partir da comparação entre os grupos com e sem pobreza multidimensional IMPM. Identificando precisamente as diferenças entre as variáveis, todas são diferentemente significativas a partir das comparações entre os grupos com pobreza multidimensional e sem pobreza multidimensional, baseados no IMPM na amostra total.

Dessa maneira, os itens são: "satisfação com sua saúde" $[F(1,731)=115,85, p<0,001]$; "satisfação com seu nível de vida" $[F(1,731)=155,08]$; "satisfação com as coisas que você tem conseguido na vida" $[F(1,731)=88,71$, $p<0,001]$; "satisfação com a sensação de estar seguro" $[F(1,731)=42,59]$; "satisfação com a comunidade" $[F(1,731)=50,52, p<0,001]$; "satisfação com a segurança a respeito do futuro" $[F(1,731)=40,95, p<0,001]$ e "satisfação com as relações com outras pessoas" $[F(1,731)=30,18$, $p<0,001]$. Também, é importante salientar que as médias mais altas e mais baixas dos itens do Índice de BEP foram as mesmas tanto para as pessoas em situação de pobreza, quanto para as fora dessa situação, nas três perspectivas de mensuração. A média mais alta foi satisfação com a relação com outras pessoas e a mais baixa foi satisfação com a segurança pessoal.

Tabela 1

Médias e desvios padrões dos itens do Índice de Bem Estar Pessoal na amostra total.

\begin{tabular}{|c|c|c|c|c|c|c|c|}
\hline & \multicolumn{7}{|c|}{ Itens do Índice de Bem Estar Pessoal } \\
\hline & Saúde & Nível de Vida & $\begin{array}{c}\text { Coisas que tem } \\
\text { conseguido na vida }\end{array}$ & Segurança & $\begin{array}{c}\text { Vincula } \\
\text { Comunitária }\end{array}$ & $\begin{array}{l}\text { Segurança com } \\
\text { o Futuro }\end{array}$ & $\begin{array}{l}\text { Relações com } \\
\text { outras pessoas }\end{array}$ \\
\hline & $M(D P)$ & $M(D P)$ & $M(D P)$ & $M(D P)$ & $M(D P)$ & $M(D P)$ & $M(D P)$ \\
\hline $\begin{array}{l}\text { Com Pobreza } \\
\text { Monetária }\end{array}$ & $7,35(2,86)$ & $7,01(2,67)$ & $7,50(2,66)$ & $6,08(3,38)$ & $7,36(2,78)$ & $6,80(2,90)$ & $8,01(2,28)$ \\
\hline $\begin{array}{l}\text { Sem Pobreza } \\
\text { Monetária }\end{array}$ & $7,58(2,47)$ & $7,54(2,18)$ & $7,87(2,27)$ & $6,51(3,00)$ & $7,73(2,48)$ & $7,07(2,64)$ & $8,31(1,95)$ \\
\hline $\begin{array}{l}\text { Com pobreza multi } \\
\text { IPM/PNUD }\end{array}$ & $7,40(2,85)$ & $7,62(2,51)$ & $8,21(2,37)$ & $6,86(3,38)$ & $7,36(2,57)$ & $7,54(2,74)$ & $8,33(2,24)$ \\
\hline $\begin{array}{l}\text { Sem Pobreza Multi } \\
\text { IPM/PNUD }\end{array}$ & $7,49(2,56)$ & $7,15(2,38)$ & $7,47(2,47)$ & $6,08(3,06)$ & $7,97(2,72)$ & $6,68(2,74)$ & $8,07(2,06)$ \\
\hline $\begin{array}{l}\text { Com pobreza multi } \\
\text { IMPM }\end{array}$ & $6,49(2,99)$ & $6,27(2,67)$ & $6,89(2,89)$ & $5,56(3,45)$ & $6,88(3,00)$ & $6,31(3,08)$ & $7,74(2,41)$ \\
\hline $\begin{array}{l}\text { Sem Pobreza Multi } \\
\text { IMPM }\end{array}$ & $8,45(1,78)$ & $8,31(1,62)$ & $8,51(1,58)$ & $7,06(2,69)$ & $8,22(2,00)$ & $7,58(2,24)$ & $8,58(1,68)$ \\
\hline
\end{tabular}




\section{DISCUSSÃo}

Sobre as análises comparativas na amostra geral, usando a perspectiva monetária, foi identificado que os mais pobres monetários têm uma média mais baixa no Índice de Bem Estar-Pessoal que aqueles menos pobres. Esse resultado é semelhante ao encontrado em uma pesquisa com 3390 adultos de seis grandes cidades na China. Foi identificado que os mais pobres monetariamente tinham médias do Índice de BEP significativamente menores do que dos outros grupos com renda mais elevada (Smyth et al., 2010).

Continuando com a perspectiva comparativa, utilizando o IPM do PNUD e também o IMPM, identifica-se que aqueles com maior situação de privação têm médias totais do Índice de bem-estar pessoal mais baixas na amostra geral. Averigua-se que a diferença entre as médias do Índice de BEP entre as pessoas em situação de pobreza e fora dessa condição tornam-se mais contrastantes, na medida em que o índice de mensuração da pobreza torna-se complexo e multidimensional.

Evidencia-se também que as pessoas em situação de pobreza têm as médias totais mais baixas do BEP, utilizando as formas multidimensionais de medição. É importante mencionar que as medidas multidimensionais, com foco na Abordagem das Capacitações, têm como objetivo ampliar o escopo do conhecimento acerca do fenômeno da pobreza, detalhando de forma multifacetada essa situação de privação (Qizilbash, \& Clark, 2005). Além disso, essa tendência da situação de pobreza impactar negativamente no bem-estar é comum em outras investigações realizadas por Hochaman e Shopeck (2013), Eastearlin (1995), Biswas-Diener e Diener (2006).

Sobre as diferenças entre os itens do BEP, comparando a amostra total a partir da perspectiva monetária, foi verificada a diferença somente entre dois itens: "satisfação com o nível de vida" e "com as coisas que tem conseguido na vida". Assim, percebe-se que a compreensão mais usual dos indivíduos frente à pobreza e à satisfação está permeada pela renda (Accorssi, 2011). Sobre essas diferenças, observa-se que esses itens de satisfação poderiam estar ligados a uma ordem monetária. Utilizando a comparação dos grupos com e sem pobreza multidimensional, a partir do IPM do PNUD, além da diferença significativa nas mesmas variáveis identificadas na comparação anterior, ocorreram diferenças significativas na sensação de estar seguro, satisfação com a comunidade e satisfação com a segurança a respeito do futuro. Assim, essas diferenças se tornam mais abrangentes a partir de um prisma de análise mais refinado e multidimensional.

Utilizando a Abordagem das Capacitações, concebe-se outras medidas de privação de liberdade para além da renda (Lelli, 2001). Dessa maneira, dimensões como saúde, educação, padrão de vida, trabalho e aspectos psicológicos da pobreza trazem uma multidimensionalidade nos impactos da pobreza. Junta-se a isso a utilização de uma medida de avaliação do bem-estar, que mensura aspectos concretos da vida (Cummins et al., 2003). Portanto, tem-se uma compreensão mais aprofundada e detalhada dos impactos da pobreza em diferentes âmbitos da vida, a partir da utilização do Índice de bem estar-pessoal.

Assim, analisando de forma específica os itens desse índice, é importante pontuar que a média do item, vinculada à satisfação de estar seguro, também é significativamente diferente entre os grupos com e sem pobreza multidimensional nas duas formas multidimensionais de mensuração. Ela também é a mais baixa em todos os grupos comparados, independentemente de serem pobres ou não pobres. Obviamente, há um fato social concreto ligado a essa realidade, que seria a grande quantidade de homicídios no Brasil (Waiselfisz, 2015), podendo repercutir na baixa satisfação com a segurança pessoal.

Em uma pesquisa realizada pelo Fórum Brasileiro de Segurança Pública (2017), a população brasileira tem um elevado sentimento de medo de sofrer diversos tipos de violência. No entanto, é importante apontar que as médias mais baixas desse item estavam situadas nas pessoas em situação de pobreza a partir dos índices de comparação multidimensionais. A incidência negativa de questões vinculadas à segurança torna-se mais evidente nos grupos mais vulneráveis. De acordo com Diprose (2007), a violência é considerada uma das dimensões perdidas da pobreza, estando totalmente imbricada nos estados de privação mais grave. Isso ocorre também porque as taxas de assassinato são mais altas, principalmente com os recortes de gênero, raça e classe social. Ou seja, aqueles que mais morrem no Brasil são jovens homens, negros e pobres (Waiselfisz, 2015). A sensação de insegurança pode afetar o grau de liberdade do indivíduo, como também sua vinculação comunitária e seus relacionamentos pessoais de maneira geral.

Corroborando com esse dado, identifica-se que há diferenças significativas da satisfação com a comunidade, utilizando o IPM do PNUD e IMPM, sendo os mais pobres em ambas as formas de mensuração com médias mais baixas. Zavaleta (2007) afirma que a pobreza pode desenvolver a diminuição da liberdade do indivíduo de ocupar espaços de socialização comunitária, causando isolamento social nas pessoas mais pobres. Obviamente, é importante entender também que aspectos vinculados à comunidade também podem ter impactos negativos no modo de vida do indivíduo, porque há comunidades, de acordo com Góis (2005), que estão estruturadas com foco na desagregação de vínculos entre os grupos mais pobres.

Relacionada a essa perspectiva relacional, também se identifica que as pessoas em situação de pobreza também têm médias baixas da satisfação com a relação com outras pessoas, quando comparadas em indivíduos fora dessa situação, a partir do IPM e do IMPM. Esse resultado pode estar relacionado aos tratamentos humilhantes geralmente 
impetrados às pessoas em situação de pobreza na sociedade, pois há uma série de estigmas negativos relacionados à identidade de pobreza (Rego, \& Pinzani, 2013). Pode-se, assim, impactar na satisfação com as relações interpessoais, pois algumas delas podem estar estruturadas em um viés discriminatório. Concebe-se que a estigmatização da pobreza pode corroborar manifestações de casos de humilhação impetrados às pessoas mais pobres, podendo gerar sofrimento e sentimentos de vergonha pela sua condição (Moura Jr., Ximenes, \& Sarriera, 2013).

No entanto, paradoxalmente, menciona-se que o índice mais elevado em todos os grupos com e sem pobreza, independente da forma mensuração, é a satisfação com as relações pessoais. Nesse caso, não há a comparação desse mesmo item entre grupos diferentes, sendo somente evidenciado seu valor absoluto em relação aos outros itens do índice de um mesmo grupo. Nas pesquisas já realizadas com o Índice de Bem-Estar Pessoal, geralmente esse item porta médias mais elevadas, demonstrando um padrão na escala utilizada (Casas et al., 2011; Casas et al., 2013). Igualmente, a sociabilidade é um dos pontos constituintes das relações humanas em geral, tendo também as pessoas em situação de pobreza relações interpessoais como centrais para sua sobrevivência, a partir da rede de solidariedades (Góis, 2005).
Portanto, identifica-se que a utilização de instrumentos de mensuração da pobreza na perspectiva multidimensional e do conceito e da medida de Bem-Estar Pessoal torna mais complexo e abrangente o panorama elucidativo do impacto da pobreza no modo de vida dos indivíduos. Quando se utiliza o IMPM, desenvolvido para este estudo como medição comparativa entre um grupo com e sem pobreza multidimensional, verifica-se que praticamente todas as variáveis têm diferenças significativas na amostra total. Utilizando essa forma de mensuração, podem-se realizar análises mais apuradas, como também estruturar políticas públicas que não tenham foco somente na transferência de renda.

As políticas de combate à pobreza também devem estar pautadas em mudanças concretas e simbólicas na realidade das pessoas mais pobres, para além da questão da renda, pois a pobreza deve ser entendida como privação de liberdade em diversos aspectos da vida, de acordo com Sen (2000). Dessa maneira, coloca-se a pessoa em situação de pobreza de forma integral no centro das ações governamentais e nas investigações, posicionado a promoção do bem-estar pessoal como meta a ser alcançada a partir dos seus múltiplos fatores constituintes.

\section{CONSIDERAÇÕES FINAIS}

Atesta-se que a pobreza, independentemente da forma de mensuração, tem um impacto negativo no Bem-Estar Pessoal. No entanto, é importante também mencionar que as medições baseadas em prerrogativas multidimensionais funcionaram como um melhor prisma de análise do fenômeno da pobreza, principalmente o IMPM, desenvolvido para este estudo. Alinhando essa estratégia de mediação com o Índice de Bem-Estar Pessoal, elabora-se um prisma de análise complexo e multifacetado sobre os impactos da pobreza.

Demonstra-se, então, a centralidade dos laços de sociabilidade nos contextos de pobreza, podendo funcionar como uma estratégia coletiva de enfrentamento desse fenômeno. Igualmente, considera-se que a realidade brasileira estaria permeada por uma cultura de violência, principalmente para o público em situação de pobreza, impactando na sensação de insegurança e privação de liberdade. Também, aponta-se que a concretude da realidade social, permeada por situações de pobreza e de violência, tem uma importância central na constituição do bem-estar pessoal. Dessa maneira, o combate à pobreza não se refere somente à transferência de renda, mas requer intervenções em níveis estruturais, culturais e simbólicos. Agradece-se à Coordenação de Aperfeiçoamento de Pessoal de Nível Superior (Capes).

\section{REFERÊNCIAS}

Abranches, S. H., Santos, W. G., \& Coimbra, M. A. (1987). Política Social e combate à pobreza. Rio de Janeiro: Jorge Zahar.

Accorssi, A. (2011). Materializações do pensamento social sobre a pobreza (Tese de Doutorado). Programa de Pós-graduação em Psicologia da Pontifícia Universidade Católica do Rio Grande do Sul, Porto Alegre, RS, Brasil.

Accorssi, A., Scarparo, H., \& Guareschi, P. (2012). A naturalização da pobreza: Reflexões sobre a formação do pensamento social. Psicologia \& Sociedade, 24(3), 536-546.

Avila, R. P., Bagolin, I. P., \& Comin, F. V. (2012). Heterogeneidade individuais versus intensidade da pobreza em Porto Alegre RS. Revista EconomiA, 13(2), 447-463.
Bagolin, I. P., \& Comim, F. V. (2008). Human Development Index (HDI) and its family of indexes: An evolving critical review. Revista de Economia, 34(2), 7-28.

Barker, C., N. Pistrang, N., \& Elliot, R. (1994). Research methods in clínical and counseling psychology. London: Willey.

Barros, R.P., Carvalho, M., \& Franco, S. (2007). A queda recente da desigualdade de renda no Brasil. Rio de Janeiro: IPEA.

Biswas-Diener, R., \& Diener, E. (2006). The subjective wellbeing of the homeless and lessons for happiness. Social Indicators Research, 76, 185-205. doi:10.1007/s11205-005-8671-9.

Brasil, Governo da República Federativa do Brasil. (2014). Decreto $n^{\circ} 8.232$, de 30 de abril de 2014. Recuperado de http://www. 
planalto.gov.br/ccivil_03/_Ato2011-2014/2014/Decreto/ D8232.htm

Casas, F., Sarriera, J. C., Abs, D., Coenders, G., Alfaro, J., Saforcada, E., \& Tonon, G. (2011). Subjective indicators of personal wellbeing among adolescents. Performance and results for different scales in latin-language speaking countries: A contribution to the international debate. Child Indicators Research, 5, 1-28. doi: 10.1007/s12187-011-9119-1

Casas, F., Fernández-Artamendi, S., Montserrat, C, Bravo, A., Bertrán, I., \& Valle, J. F. (2013). El bienestar subjetivo en la adolescencia: Estudio comparativo de dos Comunidades Autónomas en España. Anales de Psicología, 29(1), 148-158.

Cidade, E. C., Moura Jr., J. F., \& Ximenes, V. M. (2012). Implicações psicológicas da pobreza na vida do povo latino-americano. Psicologia Argumento, 30(68), 87-98.

Cidamore, A. B., \& Cattani, A. D. (2007). Produção de pobreza e desigualdade na América Latina. Porto Alegre: Tomo.

Cohen, A. G. (1996). Igualdad de qué? Sobre el bienestar, los bienes y las capacidades. In M. C. Nussbaum \& A. Sen (Orgs.), La calidad de vida (pp. 27-53). México: FCE.

Chiappero-Martinetti, E. (2000). A multidimensional assessment of well-being based on Sen's functioning approach. Rivista Internazionale di Scienze Sociali, 2, 207-239.

Chiappero-Martinetti, E., \& Moroni, S. (2007). An analytical framework for conceptualizing and re-examining the capability approach. The Journal of Socio-Economics, 26, 360-375. doi: 10.1016/j.socec.2006.12.002.

Comim, F. (2005). Capabilities and happiness: Potential synergies. Review of Social Economy, 63(2), 161-176. doi:10.1080/00346760500129871.

Comim, F. V. (2008). Operationalizing Sen's Capability Approach. In F. V. Comim, M. Qizilbash, \& S. Alkire (Eds.), The Capability Approach: Concepts, measures and application (pp. 134-160). New York: Cambridge Press.

Comim, F. V., \& Bagolin, I. P. (2002). Aspectos qualitativos da pobreza no Rio Grande do Sul. Ensaios FEE, 23, 467-490.

Comim, F. V., Bagolin, I. P., Porto Junior, S. S., Bender Filho, R., Avila, R. P., \& Picolotto, V. (2007). Indicadores de Pobreza Multidimensional e Pobreza Extrema para Porto Alegre (1 ${ }^{\mathrm{a}}$. ed.). Porto Alegre: REDE URB-AL.

Cummins, R. A., Eckersley, R., Lo, S. K., Okerstrom, E., Hunter B., \& Davern, M. (2003). Australian unity wellbeing index: Cumulative psychometric record. Report 9.0. Melbourne: Australian Centre on Quality of Life. Recuperado de http:// www.deakin.edu.au/research/acqol/instruments/PWI/ Cumulative_Psychometric_Record_Australian_data.doc.

Diener, E., Emmons, R, Larsen, J., \& Griffin, S. (1985). The satisfaction with life scale. Journal of Personality Assessment, 49(1), 71-75. doi:10.1207/s15327752jpa4901_13.

Diener, E. (2012). New findings and future directions for subjective well-being research. American Psychologist, 67(8), 590-597. doi:10.1037/a0029541.

Diprose, R. (2007). A proposal for internationally comparable indicators of violence, Missing Dimensions of the poverty. Oxford: Oxford Poverty \& Human Development Initiative, OPHI. Recuperado de http://www.ophi.org.uk/wp-content/ uploads/OPHI_wp01.pdf?39db4d

Easterlin, R. A. (1995). Will raising the incomes of all increase the happiness of all? Journal of Economic Behavior and Organization, 27, 35-47. doi:10.1016/0167-2681(95)00003-B.

Easterlin, R. A. (2000). Income and happiness: Towards a unified theory. Economic Journal, 111(473), 465-484. doi: 10.1111/1468-0297.00646

Feres, J. C., \& Villatoro, P. (2013). A viabilidade de se erradicar a pobreza: uma análise conceitual e metodológica. Cadernos de Estudos Desenvolvimento Social em Debate, 15, 10-87.

Fowler Jr., F. J. (2011). Pesquisa de levantamento. Penso: Porto Alegre.
Fórum Brasileiro de Segurança Pública. (2017). Medo da violência e o apoio ao autoritarismo no Brasil: índice de propensão ao apoio a Posições autoritárias. Texto para Debate $n^{o}$ 1. Recuperado de http://www.forumseguranca.org.br/ publicacoes/medo-da-violencia-e-o-apoio-ao-autoritarismono-brasil/

Góis, C. W. L. (2005). Psicologia Comunitária: Atividade e consciência. Fortaleza: Publicações Instituto Paulo Freire de Estudos Psicossociais.

Graham, C. (2009). Happiness around the world: The paradox of happy peasants and miserable millionaires. Oxford: Oxford University Press.

Hair, J. F., Black, W. C., Babin, B. J., Anderson, R. E., \& Tatham, R. L. (2009). Análise multivariada de dados. Porto Alegre: Bookman.

Hochman, O., \& Skopeck, N. (2013). The impact of wealth on subjective well-being: A comparison of three welfare-state regimes. Research in Social Stratification and Mobility, 34, 127-141. doi: 10.1016/j.rssm.2013.07.003.

International Wellbeing Group. (2006). Personal wellbeing indexAdult. Australian Centre on Quality of Life, Deakin University. Recuperado de http://www.deakin.edu.au/research/acqol/ instruments/wellbeing_index.htm

Instituto de Pesquisa Econômica Aplicada. (2014). Políticas Sociais Acompanhamento e Análise. Brasília: IPEA. Recuperado de http://www.ipea.gov.br/portal/images/stories/PDFs/politicas_ sociais/140930

Laderchi, C., Saith, R., \& Stewart, F. (2003). Does it matter that we don't agree on the definition of poverty? A comparison of four approaches. QEH Working Paper Series 107. Recuperado de http://ideas.repec.org/q/qeh/qehwps

Lelli, S. (2001). Factor analysis vs. Fuzzy sets theory: Assessing the influence of different techniques on sen's functioning approach. Discussion Paper Series. Leuven: Center for Economics Studies. Recuperado de http://econpapers.repec.org/paper/ wpepapers/ces0121.htm

Moura Jr., J. F., Ximenes, V. M., \& Sarriera, J. C. (2013). Práticas de discriminação às pessoas em situação de rua: Histórias de vergonha, de humilhação e de violência em Fortaleza, Brasil. Revista de Psicología, 22(2), 18-28. doi: 10.5354/07190581.2013.30850.

Moura Jr., J. F., Cidade, E. C., Ximenes, V. M., \& Sarriera, J. C. (2014). Concepções de Pobreza: Um Convite à Discussão Psicossocial. Temas em Psicologia, 22(2), 341-352. doi:10.9788/TP2014.2-06.

Moura Jr., J. F., \& Sarriera, J. C. (2017). As relações entre pobreza e bem-estar: Uma revisão sistemática. Estudos Interdisciplinares em Psicologia, 8(2), 100-125. doi: 10.5433/2236-6407.2016v8n2p100.

Nussbaum, M. C., \& Sen, A. K. (1996). La calidad de vida. México: FCE.

Programa das Nações Unidas para Desenvolvimento Humano. (1997). Informe sobre desarrolo humano. Recuperado de http:// hdr.undp.org/es/informes/mundial/idh1997/capitulos/espanol/

Programa das Nações Unidas para Desenvolvimento Humano. (2010). Relatório de Desenvolvimento Humano. Recuperado de http://www.pnud.org.br/rdh/

Qizilbash, M., \& Clark, A. (2005). The capability approach and fuzzy poverty measures: An application to the South African context. Social Indicators Research, 74, 103-139. doi:10.1007/ s11205-005-6527-y.

Rego, W., \& Pinzani, A. (2013). Vozes do Bolsa Família: Autonomia, dinheiro e cidadania. São Paulo: UNESP.

Ribeiro, P. J., \& Cummins, R. (2008). O bem-estar pessoal: Estudo de validação da versão portuguesa da escala. In: I. Leal, J. PaisRibeiro, I. Silva, \& S. Marques (Eds.), Actas do $7^{\circ}$ congresso nacional de psicologia da saúde (pp. 505-508). Lisboa: ISPA. 
Robson, C. (1993). Real world research: A resource for social sciences and practioner-researcher. Oxford: Blackwell.

Samman, E. (2007). Psychological and Subjective Wellbeing: A proposal for a internationally comparable indicators. Working Paper Series 05. Oxford: Oxford Poverty \& Human Development Initiative, OPHI. Recuperado de http://www. ophi.org.uk/wp-content/uploads/OPHI-wp05.pdf

Sen, A. K. (1980). Equality of What? In S. M. Mcmurrin (Ed.), Tanner lectures on human values (pp. 195-220). Cambridge: Cambridge University Press.

Sen, A. K. (1996). Capacidad y bienestar. In M. C. Nussbaum \& A. K. Sen (Eds.), La calidad de vida (pp. 54-83). México: FCE.

Sen, A. K. (2000). Desenvolvimento como liberdade. São Paulo: Companhia das Letras.
Smyth, R., Nielsen, I., \& Zhai, Q. (2010). Personal Well-being in Urban China. Social Indicators Research, 95, 231-251. doi:10.1007/s11205-009-9457-2.

Waiselfisz, J. J. (2015). Mapa da Violência 2014: Jovens do Brasil. FLASCO: Rio de Janeiro. Recuperado de http://www. mapadaviolencia.org.br/pdf2014/Mapa2014_JovensBrasil_ Preliminar.pdf

Zavaleta, D. R. (2007). The ability to go about without shame: A proposal for internationally comparable indicators. Working Paper 03 OPHI.Oxford: Oxford Poverty \& Human Development Initiative, OPHI. Recuperado de http://www. ophi.org.uk/working-paper-number-03/ 